\title{
Über das Morphin
}

von

Zd. H. Skraup und D. Wiegmann.

Aus dem chemischen Institut der k. k. Universität Graz.

(Mit 1 Textfigur.)

(Vorgelegt in der Sitzung am 14. Februar 1889.)

Untersuchungen von Gerichten, Schrötter und 0 . Fischer haben mit Sicherheit ergeben, dass das Morphin $\mathrm{C}_{17} \mathrm{H}_{19} \mathrm{NO}_{3}$ ein Derivat des Phenanthrens $\mathrm{C}_{14} \mathrm{H}_{10}$ ist. Über die ausserhalb des Phenanthrenringes anzunehmenden drei Kohlenstoffatome und weiter über die Bindung des Morphinstickstoffes sind die Nachrichten weit weniger bestimmt. Abgesehen von einigen Beobachtungen, dass bei energischen Processen aus dem Morphin Methylamin abgespalten wird, dessen Auftreten natürlich in der versehiedensten Art erklärt werden kann, liegen Angaben über den Zerfall von Alkylderivaten des Morphins vor, die mebr verwirren als aufklären. ${ }^{4}$

Denn während v. Gerichten und Schrötter ${ }^{2}$ beobachtet haben, dass das Methylmorphäthinmethylhydroxyd beim Erhitzen neben einem Phenanthrenderivat $\mathrm{C}_{15} \mathrm{H}_{10} \mathrm{O}_{2}$, als stickstoffhältiges Spaltungsproduct Methyläthylpropylamin liefert, dessen Propylgruppe doch nur dem Morphinmolekül entstammen kann, findet Hesse $\mathrm{e}^{3}$ dass das Methylmorphmethinmethyllydroxyd Trimetbylamin, und nicht wie analog den Beobachtungen der erst-

1 Vorliegende Untersuchung war beendet, als Knorr's Arbeit: Zur Kenntniss des Morphin's (Berl. Ber. 22,181) erschienen ist. Wir kommen auf dieselbe zum Schlusse unserer Mittheilung zurïck.

2 Berl. Ber. 15, 1484, 2179.

3 Li ebig's Ananlen 222, 232. 
genannten Cbemiker zu erwarteñ gewesen wäre Dimethylpropylamin abspaltet.

Der erstangeführte Process führt zu der Annahme, dass der Morphinstickstoff mit der Propylgruppe verbunden sei, der zweite zu dem Schlusse, dass am Stickstoff Methyl angelagert ist.

Eine Entscheidung auf anderem Wege war umso wïnschenswerther, als v. Gerichten und Schrötter blos die Bestimmung des Platingehaltes im Chloroplatinat ihres flüchtigen Spaltungsproductes beschreiben und Hess e garkeine analy tischen Daten veröffentlicht hat. Bei solchen neuen Versuchen erschien es angezeigt, rom Morphin selbst auszugehen und damit jenen Irrungen auszuweichen, die durch Austausch und Platzwechsel von Alkylgruppen herbeigeführt werden können.

\section{Einwirkung von Alkalien auf Morphin.}

Wenn Morphin mit alkoholischer Kalilange, mit Natriumäthylat oder Natriumamylat hoch erhitzt wird, spaltet es ein flüchtiges Amin $a b$, und daneben einen phenolartigen nicht basischen Körper. Wäbrend es gelungen ist, Verhältnisse zu finden, unter welchen die Ausbeute an dem Amin wenig zu wünschen übrig lässt, waren wir, was das Phenol betrifft, weniger gltïcklich und haben wir deshalb seine Untersuchung vorläufig aufgegeben.

Von den vielen Vorversuchen sei nur jener kurz erwähnt, bei welchem Morphin mit einer Lösung von Natrium in Amylalkohol erhitzt wurde, weil unter diesen Bedingungen ganz erhebliche Mengen von Piperidin entstanden, die offenbar von dem Pyridin abstammen, das nach Haitinger in jedem käufliehen Amylalkohol vorkommt. Da Amylalkohol bei der Reduction stickstoffhältiger Verbindungen mit Natrium bäufig verwendet wird, glauben wir unsere Erfahrung, so selbstverständlich sie auch ist, nicht verschweigen zu sollen.

Die vorhin erwähnte flüchtige Base entsteht am reichlichsten, wenn Morphin mit der 10 bis 15fachen Menge einer 20 Pere. Lösung von Ätzkali in Alkohol 4 bis 6 Stunden auf $180^{\circ}$ erhitzt wird. Das Füllen und Entleeren der Erhitzungsröhren haben wir in einer Lenchtgasatmosphäre vorgenommen, ebenso wurde der 
Röhreninhalt durch Lenchtgas in etwas mehr als die berechnete Menge verdünnter Schwefelsäure übergedriickt; trotzdem war die Flüssigkeit immer dunkelbraun gefärbt; kohlige Reste, gasige Zersetzungsproducte oder andere Anzeichen einer totalen Zersetzung traten jedoch nicht auf.

Nach dem Eintritt in verdünnte Schwefelsäure scheiden sich reichlich lichtbräunliche Flocken eines leicht zersetzlichen Körpers $\boldsymbol{A} \mathrm{ab}$, der besonders im feuchten Zustand nachdunkelt und darum sofort entweder wie weiter beschrieben, gereinigt oder nach sorgfältigem Waschen mit Wasser im Vacuum getrocknet wird.

Das bräunliche Filtrat von dieser festen Abscheidung gab an $\ddot{A}$ ther nur sehr geringe Mengen einer nicht krystallisirenden Substanz ab, ebenso an andere Lösungsmittel; es wurde sofort mit Soda übersättigt und im Wasserdampfstrom destillirt, bis die Dämpfe nicht mehr alkalisch reagirten. Die Destillate flossen in überschïssige titrirte Salzsäure, so dass nach Beendigung der Destillation durch Titration die Menge des Morphinstickstoffs der in flüchtige Producte übergegangen war, ermittelt werden konnte.

Die ersten Versuche haben wir in Glasröhren ausgeführt, später eiserne, sogenannte Druckrohre benützt, die an einem Ende zusammengeschweisst am andern offenen mit einem Schraubengewinde und einem scharf zulaufenden Rande versehen waren. Der Verschluss erfolgte durch Aufschrauben eines kurzen Rohrstückes, das halb mit Blei ausgegossen, so dicht in den scharfen Rand einschnitt, dass auch nach mehrstündigem Erhitzen eine Gewichtabnahme nicht eintrat. Es empfiehlt sich das Blei vor jedesmaligem Gebrauch umzuschmelzen und die Oberfläche desselben blank zu putzen.

Die Substanz $A$ löst sich in indifferenten Lösungsmitteln nur wenig auf; Alkalien lösen sie leicht, ebenso concentrirte Säuren, verdünnte Säuren schwierig. Die alkalischeLösung dunkelt an der Luft sehr rasch nach. Nachdem alle Versuche, den Körper aus Lösungsmitteln krystallisirt zu erhalten, oder ihn in ein leicht krystallisirendes Derivat umzuwandeln, entweder völlig fehlschlugen oder wegen äusserst schlechten Ausbeuten aufgegeben werden mussten, haben wir seine Reinigung durch partielle Fällung vorgenommen. 
$\mathrm{Zu}$ diesem Behufe wird die fein zerriebene Substanz in wässerigem Alkohol suspendirt und in der Wärme Natriumbisulfit zugefügt, bis auf geringe dunkle Reste alles gelöst ist. Die filtrirtelösung wird mitWasser partiell gefällt, die erhaltenen Niederschläge durch nochmaliges Lösen in verdünntem Weingeist unterZusatz vonBisulfit, und Ausfällen mit Wasser weiter gereinigt. So gelingt es unter grossen Verlusten ans den letzten fast ungefärbten Lösungen durch Zusatz von viel Wasser fast reinweisse mikrokrystallinische Flocken niederzuschlagen, die weniger empfindlich wie die rohe Snbstanz, doch noch immer sehr zersetzlich sind and schon beim Trocknen über Schwefelsäure röthlich bis bräunlich verfärbt werden. Die Mutterlaugen der Fällung durch Destillation im Vaccuum von Alkohol befreit, scheiden dieselbe Substanz ab, wie eine freilich nicht scharf stimmende Analyse und die Übereinstimmung aller Eigensehaften ergeben hat.

$0 \cdot 1802 g$ über Schwefelsäure bis zum constanten Gewicht getrocknet gaben $0.4196 \mathrm{~g} \mathrm{CO}_{2}$ und $0.0966 \mathrm{~g} \mathrm{H}_{2} \mathrm{O}$.

$0 \cdot 2348 \mathrm{~g}$ gaben $7 \cdot 3 \mathrm{~cm}^{3} \mathrm{~N}$ bei 240 und $733 \cdot 6 \mathrm{~mm}$

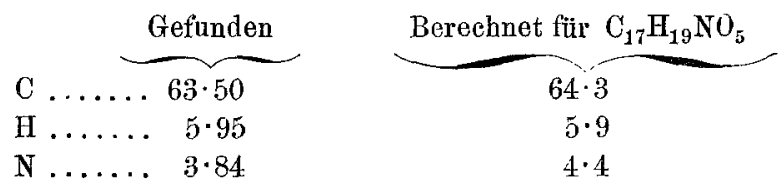

Die Substanz hat also die Zusammensetzung eines Dioxymorphins, ob sie thatsächlich ein so einfaches Morphinderivat ist, kann umso weniger entschieden werden, als alle Versuche sie genauer zu untersuchen resnltatslos blieben. Charakteristisch ist ihr Verhalten gegen Oxydationsmittel, welehe bei vorsichtiger Einwirkung prächtig roth färben. Wird z. B. die Substanz in heissem Alkohol suspendirt und Eisenchlorid zugefugt, so tritt bei gelindem Erwärmen unter gleichzeitiger Dunkelrothfärbung Lösung ein und Wasser schlägt dann ein rothes Pulver nieder, das frisch bereitet in Alkohol sehr leicht löslich ist und von Natriumbisulfit entfärbt wird; schon nach kurzer Zeit löst Alkohol aber nar theilweise und das sulfit liefert eine bräunliche Substanz. 


\section{Flüchtiges Amin aus Morphin.}

Durch die früher erwähnte Titration des alkalischen Destillates kamen wir zu der aufälligen Beobachtung, dass bloss etwa 50 Perc. des Morphins in flïchtige Amine übergehen, auch wenn die Dauer der Einwirkung verlängert und die Concentration der Kalilauge erhöht wird. In der Mehrzahl der Versuche fanden wir 46-49 Perc., im Durchschnitt aller Bestimmungen 40 Perc., bloss eine Bestimmung ergab 57 Perc. was auch einem Versuchsfehler zugeschrieben werden kann. Um jeden Irrthum auszuschliessen, sind scbliesslich die eisernen Rohre nicht mehr, dafür Verbrennungsrohre verwendet worden, ohne aber andere Zahlen zu erbalten. Unter allen Umstënden waren reichliche Mengen von einem stickstoffhältigen Körper vorhanden, der die Eigenschaften des Dioxymorphins besass.

Das mit Salzsäure neutralisirte Destillat färbt sich beim Eindampfen gelblich bis bräunlich, bei genïgender Concentration erstarrt es zu unregelmässig geformten tafelartigen Krystallen.

Ein Theil mit überschüssigem Platinchlorid vermischt setzte nach kurzer Zeit gelbrothe, einzeln angeordnete Prismen an, die vollständig homogen aussahen, dieselben Krystalle lieferte die Mutterlauge bei weiterem Einengen.

Sie verwittern nicht, sind in kaltem Wasser wenig, in heissem sehr leicht löslich, ziemlich löslich in verdünntem Weingeist, nicht in Alkohol und Äther. Das so gewonnene Platinsalz schmilzt bei 205, ganz reine Substanz, wie sie aus der durch Destillation gereinigten Base erhalten wurde, aber bei $207-208^{\circ}$.

Analyse 1 ist mit dem direct dargestellten, Analyse 2 mit dem aus destillirten Amin gewonnenen Salz ausgeführt.

1. $0.2257 g$ gaben $0.1125 g \mathrm{CO}_{2}$ und $0.0832 g \mathrm{H}_{2} \mathrm{O}$ sowie $0.0847 g \mathrm{Pt}$.

2. $0 \cdot 2138 g$ gaben $0.0773 g \mathrm{Pt}$.

\begin{tabular}{|c|c|c|c|}
\hline \multicolumn{3}{|c|}{ Gefunden } & \multirow{2}{*}{$\begin{array}{c}\begin{array}{c}\text { Berechnet für } \\
\left(\mathrm{C}_{3} \mathrm{H}_{9} \mathrm{~N}\right)_{2} \mathrm{H}_{2} \mathrm{PtCl} l_{6}\end{array} \\
\end{array}$} \\
\hline & & II & \\
\hline $\mathrm{c} \ldots \ldots$ & $13 \cdot 59$ & - & $13 \cdot 62$ \\
\hline $\mathrm{H} \ldots \ldots$ & $4 \cdot 11$ & - & $3 \cdot 78$ \\
\hline $\mathrm{Pt} .$. & $37 \cdot 52$ & $37 \cdot 15$ & $36 \cdot 93$ \\
\hline
\end{tabular}

Die flichtige Base hat demnach die Zusammensetzung $\mathrm{C}_{3} \mathrm{H}_{9} \mathrm{~N}$, die Analyse des nicht weiter gereinigten Präparates zeigt weiter dass Amine von anderem Kohlenstoffgehalte nur in Spuren beigemengt sein können. 
Die Hauptmenge des Chlorbydrates mit höchst concentrirter Kalilauge zerlegt, schied eine leichte wasserhelle Flüssigkeit ron intensiv aminartigem doch von dem des Trimethylamins verschiedenem Geruch ab. Sie wurde mit festem Ätzkali sorgfältig getrocknet, und ging dann constant zwischen $34-35$ uncorr. itber. Nur wenige Tropfen destillirten noch bis $37^{\circ}$.

Der Siedepunkt des Amins schliesst das normale Propylamin aus, ebenso das Trimethylamin, liegt aber sehr nahe jenem des Isopropylamins für welches Gautier 31.5 als Siedetemperatur angibt.

Die Fraction 34-35 mit Wasser vermischt mit Salzsäure neutralisirt und am Wasserbade, schliesslich ïber Schwefelsäure concentrirt gab wasserhelle Krystalle die rasch auf trockenes Porzellan gestrichen und allsogleich wieder uber Scbwefelsäure gestellt wurden um sie von der beim Concentriren wieder gelb gewordenen Mutterlange zu befreien.

Nach mehrtägigem Stehen zeigten sie den Schmelzpunkt 132, während Gautier als Verflüssignngspunkt des Isopropylaminsalzes 139.5 angibt. Da beim Einfiillen in die Capillarröhrehen merklich Wasser angezogen nnd deshalb der Schmelzpunkt leicht zu niedrig gefunden wird, haben wir unter häufigem Verreiben über Schwefesäure getrocknetes Salz möglichst rasch in mebrere Röhrchen eingefüllt, diese wieder über Schwefelsäure ins Vacuum gestellt, nach etwa acht Tagen mit einem derseiben die Schmelzpunktsbestimmung ausgeführt und letztere in Zeiträumen von mehreren Tagen bis Wochen wiederholt. Schon bei der zweiten Bestimmung verflüssigte sich das Salz scharf bei $133^{\circ}$ und dasselbe Resultat gaben alle späteren Wiederholungen.

Die Eigenschaften unseres Amins weichen von jenen des Isopropylamins zu sebr ab, um ohne weiters eine Identification zuzulassen. Hierbei ist noch zu erwägen, dass das vierte Isomere, der Formel $\mathrm{C}_{3} \mathrm{H}_{9} \mathrm{~N}$, das Äthylmethylamin noch nicht dargestellt ist, möglicherweise dem Isopropylamin in den Eigenschaften sehr nahesteht und deshalb das Amin aus Morphin auch das unbekannte Äthylmethylamin sein könne. Dazu kommit noch, dass die Morphinbase die Isonitrilrealition äusserst schwach gab, die Senfölreaktion ganz versagte. 
Es blieb deshalb nichts übrig als das Äthylmethylamin synthetisch darznstellen. Um den Vergleich zu erleichtern, haben wir das Amin aus Morphin noch in das Golddoppelsalz und in das saure Oxalat verwandelt. In beiden Fällen diente durch Destillation gereinigte Base.

Gold doppelsalz. Dasselbe fällt aus der Lösung des Chlorhydrates nach Zusatz der berechneten Menge Goldchlorid in zarten langen Nadeln aus, die in kaltem Wasser schwierig löslich sind und bei 179-180 schmelzen.

$0 \cdot 2189 \mathrm{~g}$ gaben $0.1077 \mathrm{~g} \mathrm{Au}$

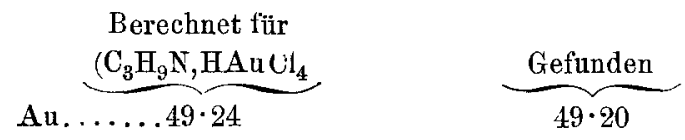

Saures Oxalat. Es bildet weisse haarfeine Nadeln, die oft zu glänzenden Schuppen verwachsen sind, die in der Hitze in Wasser und Alkohol leicht, in der Kälte schwierig löslich sind, und wiederholt aus absolutem Alkohol unkrystallisirt bei $154-155^{\circ}$ schmelzen.

$0.2222 g$ gaben $0.3238 \mathrm{~g} \mathrm{Co}_{2}$ und $0.1450 \mathrm{~g} \mathrm{H}_{2} \mathrm{O}$

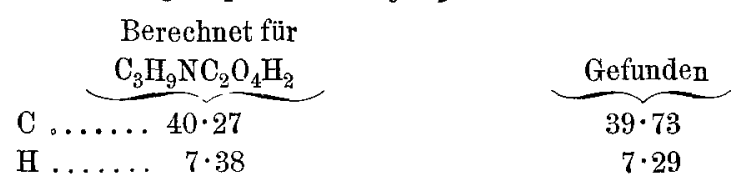

\section{Darstellung des Äthylmethylamins.}

Das gesuchte Äthylmethylamin bereiteten wir durch Einwirkung von Jodmethyl auf ein Gemisch von käuflicher 30 Perc.

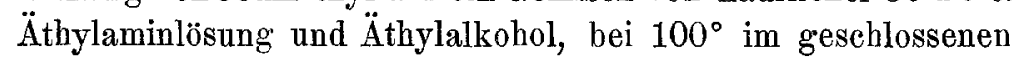
Rohr. Nach dreistiundigem Erhitzen wurden die freien Amine mit Wasserdampf abdestillirt, der Rückstand bis zur Krystallisation gedampft, mit höchst concentrirter Kalilange zerlegt und abermals Wasserdampf eingeleitet, welcher die vorher als jodwasserstoffsauren Salze gebundenen Basen übertrieb.

Im Kolben blieb neben Jodkalium ein nach dem Erkalten krystallisirendes $\ddot{O l}$, das mechanisch von ersteren getrennt, durch wiederholtes Umkrystallisiren aus Alkohol gereinigt wurde: Es ist in heissem Alkohol leicht, in kaltem schwierig, ziemlich leicht in Wasser löslich, Jodkalium fällt es aus Wasser ans. 
Die Krystalle, weisse lange Nadeln, lassen sich vorsichtig erhitzt, unverändert sublimiren, ohne vorher zu schmelzen, grössere Mengen werden aber theilweise zerlegt, indem sich Jod abscheidet und Amingeruch auftritt; sie sind krystallwasserfrei und der Analyse nach das Äthyltrimethylammoniumjodid. $0.3035 \mathrm{~g}$ Substanz bei $100^{\circ}$ getrocknet gaben $0.3290 \mathrm{~g} \mathrm{Ag} \mathrm{I}$

$$
\underbrace{\begin{array}{l}
\text { Berechnet für } \\
\mathrm{C}_{3} \mathrm{H}_{5}\left(\mathrm{CH}_{3}\right)_{3} \mathrm{NI}
\end{array}}_{\mathrm{I} \ldots \ldots 58 \cdot 9} \quad \underbrace{\text { Gefunden }}_{59 \cdot 0}
$$

Die durch Ätzkali frei gewordenen Basen haben wir nach dem Verfahren von Duvillier und Buisin e ${ }^{1}$ getrennt.

Die ziemlich concentrirte wässerige Lösung der Amine mit der berechneten Menge Oxalsäureäther $(50 \mathrm{~g})$ geschüttelt, schied nach kurzer Zeit weisse Nädelchen ab, deren Menge nach einigen Stunden nicht mehr zunahm. Sie waren Diäthyloxamid das aus Wasser umkrystallisirt bei $178^{\circ}$ schmolz.

$0 \cdot 2945 \mathrm{~g}$ gaben $52 \cdot 4 \mathrm{~cm}^{3} \mathrm{~N}$ bei $22 \cdot 5^{\circ}$ und $732 \mathrm{~mm}$

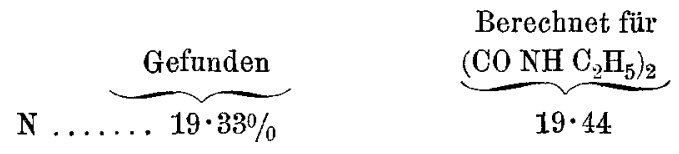

Das Filtrat von Diäthyloxamid mit Wasserdampf destillirt lieferte eigenthümlicherweise kein alkalisches Destillat, sondern bloss kleine Mengen Oxalsäureäther; es scheint also ein tertiäres Amin höchstens in Spuren entstanden zu sein.

Nach dem Erkalten hatten sich abermals Nädelchen des Diäthyloxamids abgesetzt und weitere Mengen desselben konnten noch mit Ather extrahirt werden.

Auch die mit $\ddot{A}$ ther ausgezogene Substanz ist analysirt worden. $0 \cdot 1400 \mathrm{~g}$ gaben $0 \cdot 2520 \mathrm{~g} \mathrm{CO} \mathrm{O}_{2}$ und $0 \cdot 1038 g \mathrm{H}_{2} \mathrm{O}$

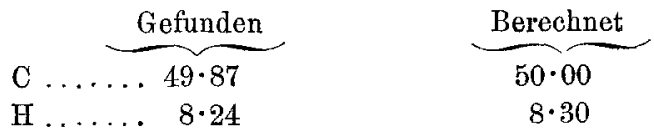

1 Ann. chim. phys. (V) 23, 289. 
Die rom Oxamid völlig befreite Lösung haben wir gemäss dem Vorschlag von Duvillier und Buisine stark verdünnt und anhaltend gekocht, um die vorhandenen Äther zu verseifen. Nach abermaliger Concentration erstarrte die Flüssigkeit allmälig zu einem Brei glänzender Blättchen, die von der zähen Mutterlauge nur derart zu trennen waren, dass sie zuerst, so gut es ging, auf der Saugpumpe filtrirt, dann öfter mit wenig wässerigem Alkohol angerieben und wieder abgesaugt wurden.

Sie schmelzen nach öfterem Umkrystallisiren aus Alkohol scharf bei 113-114, sind in Wasser sehr leicht, schwieriger in Alkohol löslich und nach Zusammensetzung und Verhalten zweifellos Saures Oxalsaures Athylamin. $0 \cdot 2420 g$ gaben $0 \cdot 3114 g \mathrm{CO}_{2}$ und $0 \cdot 1505 g \mathrm{H}_{2} \mathrm{O}$
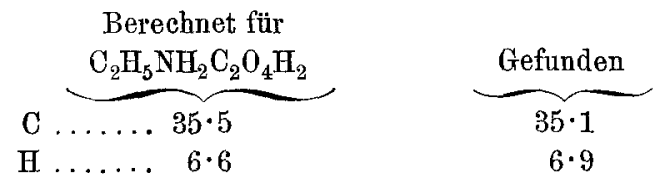

$0.5 \mathrm{~g}$ in Wasser gelöst, nach Zusatz von etwas überschüssigem Ätznatron destillirt, hierauf in bekannter Art mit Chlorcalcium gefällt, gaben $41 \cdot 5 \%$ CaO, berechnet $41 \cdot 48$.

Das durch Natronlauge freigemachte Amin der Analyse 2 in das Chloroplatinat verwandelt zeigte die dem Äthylaminsalz zukommende Zusammensetzung.

$0.2034 g$ hinterliessen $0.0770 \mathrm{~g} \mathrm{Pt}$

Berechnet für
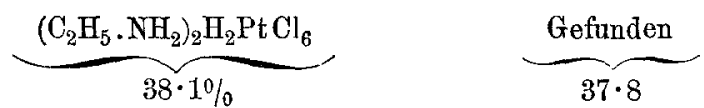

Aus der Mutterlauge des Äthylaminoxalats fallen nach weiterem Einengen weisse Nadeln aus, die sich sowohl durch ibre Form als durch den höheren Schmelzpunkt von der schon beschriebenen ersten Krystallisation auffallend unterscheiden Nach öfterem Umkrystallisiren aus Alkohol steigt der Schmelzpunkt bis $154^{\circ}$ und erhöht sich dann nicht mehr. Die Nadeln sind in Wasser und verdünntem Alkohol leicht, in absolutem Alkohol schwieriger löslich, vorsichtig erhitzt lassen sie sich anscheinend nnverändert sublimiren. 
Die Analyse zeigte, dass das saure Oxalat des gesuchten Äthylmethylamins vorlag.

$0.2428 g$ gaben $0.3626 g \mathrm{CO}_{2}$ und $0.1606 g \mathrm{H}_{2} \mathrm{O}$

Berechnet für
C . . . Gefunden
$\underbrace{\left(\mathrm{C}_{2} \mathrm{H}_{5}\right)\left(\mathrm{CH}_{3}\right) \mathrm{NH} . \mathrm{C}_{2} \mathrm{O}_{4} \mathrm{H}_{2}}_{\begin{array}{r}40 \cdot 27 \\ 7 \cdot 38\end{array}}$
H ...... $7 \cdot 34$

$0 \cdot 4011 \mathrm{~g}$ lieferten in derselben Art behandelt wie beim $\ddot{A}$ thylaminoxalat beschrieben ist: $0 \cdot 1465 g \mathrm{CaO}$

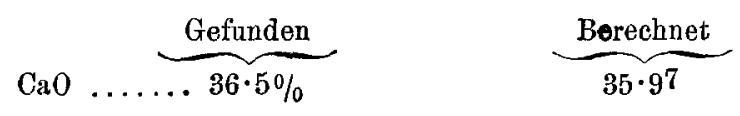

Das aus dem Oxalat abgeschiedene Amin lieferte ein Chlorhydrat das in Tafeln krystallisirt, die jenen des Salzes aus Morphin sehr ähnlich waren, deren Schmelzpunkt aber constant niederer, bei 126-127 lag. Sie waren etwas gelb gefärbt, die geringe Menge verhinderte eine weitere Reinigung.

Auf Zusatz von Goldchlorid gab das Chlorhydrat des Äthylmethylamins einen dichten Niederschlag, der in kaltem Wasser schwierig löslich, aus heissem umkrystallisirt werden konnte und dann lange Nadeln bildete, die dem Goldsalze aus Morhpin zum Verwechseln ähnlich, gerade so wie dieses bei 179-180 sich verflüssigten.

Aus dem Rest der Substanz haben wir das Chloroplatinat dargestellt das in Ansehen und Lösliehkeitsverhältnissen mit dem aus Morphin identisch ist und ebenso wie letzteres bei $208^{\circ}$ schmilzt.

$0 \cdot 2529 g$ gaben $0 \cdot 0940 g \mathrm{Pt}, 0 \cdot 1268 g \mathrm{CO}_{2}$ und $0.0886 g \mathrm{H}_{2} \mathrm{O}$

\begin{tabular}{|c|c|}
\hline Gefunden & $\begin{array}{c}\begin{array}{c}\text { Berechnet für } \\
\left(\mathrm{C}_{3} \mathrm{H}_{9} \mathrm{~N}\right)_{2} \mathrm{H}_{2} \mathrm{Pt} \mathrm{Cl}_{6}\end{array} \\
\end{array}$ \\
\hline C $\ldots \ldots, 13 \cdot 67$ & $13 \cdot 62$ \\
\hline H . . . . $3 \cdot 89$ & $3 \cdot 78$ \\
\hline Pt $\ldots \ldots .37 \cdot 17$ & 37.16 \\
\hline
\end{tabular}

Herr K. Lippitsch hat die Verbindung krystallographisch untersucht und gleichzeitig auch Messungen mit dem Chloroplatinat der Morphinbase ausgeführt. Es hat sich herausgestellt, 
dass die beiden Substanzen äusserst ähnlich, aber nicht völlig gleich krystallisiren. Er theilt uns mit:

"Krystalle, lang gestreckt, nadelförmig, nach der optischen Untersuchung rhombisch. Es treten auf $\infty P, \infty P \infty, P \varpi ; \infty P$ immer schlecht entwickelt.

Äthyl-Methylamin-Platinsalz

$$
\begin{aligned}
& \infty P: \infty P=125^{\circ} 30^{\prime} \\
& \infty P: \infty P \breve{\infty}=11615 \\
& P \breve{\infty}: P \check{\infty}=11654 \\
& \check{\infty} \check{\infty}: P \check{\infty}=12120
\end{aligned}
$$$$
a: b: c=0 \cdot 5141: 1=0 \cdot 6128
$$

Da die beiden Verbindungen dieselben Formen aufweisen, und nur die Differenz des einen Winkels von $2^{\circ}$ vorliegt, sind sie wohl krystallographisch identisch, umsomehr als das Chlorplatinat des

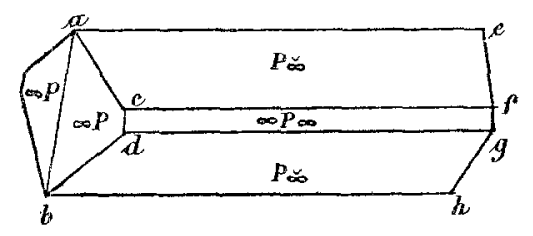
Isopropylamins, das noch in Betracht kommen könnte, nicht nur ganz andere Formen, sondern auch ein ganz anderes Axenverhältnis wie jenes der Morphinbase besitzt."

Da somit die Verbindungen des synthetischen Äthylmethylamins nur sehr geringfügig von jenen der flüchtigen Morphinbase abweichen, letztere aber von den beiden Propylaminen und dem Trimethylamin sich sehr wesentlich unterscheidet, kann über ibre Natur weiter kein Zweifel herrschen.

Auch noch auf anderem Wege gelang es das Amin aus Morphin als secundäre Base zu erkennen; es geht nähmlich in methylalkoholischer Lösung mit überschüssigem Jodmethyl erhitzt, zum grössten Theil in Äthyltrimethylammoniumjodid uiber, das alle Eigenschaften des Jodids aufweist, wie wir es als Nebenproduct der Darstellung von Äthylmethylamin erhalten haben. Die Isolirung geschah nach bekannten Methoden, erwähnt sei, dass zur Abscheidung der tertiären Basen Kaliumcarbonat genommen werden muss, da Ätzkali das quaternäre Jodid angreift. 
$0 \cdot 2164 \mathrm{~g}$ gaben $0 \cdot 2359 \mathrm{~g} \mathrm{Ag} \mathrm{I}$

$$
\underbrace{\begin{array}{c}
\text { Berechnet für } \\
\left(\mathrm{C}_{2} \mathrm{H}_{5}\right)\left(\mathrm{CH}_{3}\right)_{3} \mathrm{NI}
\end{array}}_{\text {I } \ldots \ldots 58.97} \underbrace{\text { Gefunden }}_{58.87}
$$

Nach Beendigung dieser Versuche konnten wir die letzter Zweifel durch einen Vergleich mit reinem Isopropylamin beseitigen, von dem Herr Tafel uns fast ein Gramm zu überlassen die Güte hatte, wofïr wir ihm auch an dieser Stelle bestens danken.

Es zeigte sich, dass das Isopropylamin die Isonitrilreaction ganz deutlich gibt, dass sein Chloroplatinat in schmalen zusammengewachsenen Blättern krystallisirt, die ganz verschieden von den vereinzelt ausgebildeten Prismen der Morphinbase aussehen, und bei 227 - $228^{\circ}$ schmelzen; das salzsaure Isopropylamin kurze Zeit bei $100^{\circ}$ getrocknet verflüssigte sich bei $153-55^{\circ}$, das Goldsalz aus dem bei $100^{\circ}$ getrockneten Chlorhydrat in Form flächenreicher gelber Täfelchen gewonnen bei $72-73^{\circ}$. Nach all dem ist die aus Morphin beim Erhitzen mit alkoholischer Kalilauge entstehende Base bestimmt Äthylmethylamin.

Da mit letzterem die ganze Reihe der Structur- verschiedenen Isomeren der Formel $\mathrm{C}_{3} \mathrm{H}_{9} \mathrm{~N}$ bekannt ist, erscheint eineZusammenstellung ihrer charakteristischen Merkmale nicht iiberflüssig.

\begin{tabular}{|c|c|c|c|c|}
\hline & Propylamin & $\begin{array}{l}\text { Isopropyl- } \\
\text { amin }\end{array}$ & $\begin{array}{c}\text { Äth ylmethyl- } \\
\text { amin }\end{array}$ & $\begin{array}{l}\text { Trimethyl- } \\
\text { amin }\end{array}$ \\
\hline Siedepunkt & $49^{\circ}$ & $31 \cdot 5^{\circ}$ & $\begin{array}{l}34-35^{\circ} \\
\text { S. u. W. }\end{array}$ & $9 \cdot 3^{\circ}$ \\
\hline Chlorhydrat. & Blätter? & Wïrfèl & Tafeln & Prismen \\
\hline Schmzp. & $155-158$ & $\left\{\begin{array}{l}139.5 \\
153.55\end{array}\right\}$ S.u. W. & $\begin{array}{c}133^{\circ} \\
\text { S. u. W. }\end{array}$ & ? \\
\hline Platinsalz & Nadeln & $\begin{array}{c}\text { schmale } \\
\text { Blätter }\end{array}$ & Nadeln & $\begin{array}{l}\text { derbe } \\
\text { Tafeln }\end{array}$ \\
\hline Schmzp. & $\begin{array}{l}214^{\circ} \\
\text { S. u. W. }\end{array}$ & $\begin{array}{l}227-228^{\circ} \\
\text { S. u. W. }\end{array}$ & $\begin{array}{l}208^{\circ} \\
\text { S. u. W. }\end{array}$ & $\begin{array}{l}240-45^{\circ} \\
\mathrm{Kn} \text { orr }\end{array}$ \\
\hline Goldsalz & lange Nadeln & Täfelchen & lange Nadeln & $\begin{array}{l}\text { Krystall- } \\
\text { körner }\end{array}$ \\
\hline Schmzp. & $\begin{array}{c}169^{\circ} \\
\text { S. u. W, }\end{array}$ & $\begin{array}{l}72-73^{\circ} \\
\text { S. u. W. }\end{array}$ & $\begin{array}{c}179-180^{\circ} \\
\text { S. и. W. }\end{array}$ & $253^{\circ} \mathrm{Kn}$ orr \\
\hline
\end{tabular}

Tabelle I. 
In dieser Zusammenstellung sind die von uns ermittelten Daten mit S. und W. bezeichnet.

\section{Nachschrift.}

Die beschriebenen Versuche waren mit Ausnahme der mit dem Isopropylamin ansgeführten beendet, als uns die Mittheilung von L. Knorr zur Kenntniss des Morphins zukam, welche mit unseren schwer vereinbare Ergebnisse enthält.

Denn während nach unseren Beobachtungen der Morphinstickstoff zwei Alkylgruppen bindet, und daher entgegengesetzt zu der bisherigen Annahme bloss mit einer Valenz mit dem Phenantrenreste verbunden sein kann, findet Knorr Thatsachen, welche, wie wir gern zugeben, ebenso bestimmt dafür sprechen, dass am Morphinstickstoff nur eine Alkylgruppe und zwar Methyl gebunden ist.

Die Entstehung von Trimethylamin aus Methylmorphimethinmethylhydroxyd liesse sich mit unserer Annahme allerdings unter der Voraussetzung in Zusammenhang bringen, dass die $\ddot{A}$ thylgruppe als Äthylen abgespalten wird, wie Hesse ${ }^{1}$ schon bei anderer Gelegenheit erwähnt. Dann bleibt es aber noch immer unverständlich, dass Knorr aus dem Methylmorphimethin blos Dimethylamin und nicht, wie nach unseren Beobachtungen wahrscheinlich wäre, Dimethyläthylamin abgespalten hat.

Wir haben einen Augenblick an einen groben Versuchsfehle $r$ unserer Untersuchung gedacht und sie deshalb in den wichtigsten Theilen wiederholt, doch ohne die so eringste Differenz auffinden zu können.

Wir erhielten das Äthylmethylamin und ohne merkliche Mengen anderer Amine auch dann, als wir Alkohol benützten, der zuvor uiber Oxalsäure destillirt wurde, (1), wir erhielten es auch bei Einwirkung von methylalkoholischer Kalilauge, (2), es entstand auch beim Erhitzen von weingeistigem Ätzkali und Codein. (3) In allen drei Versuchen haben wir die Chloroplatinate analysirt, letztere verflüssigten sich bei 208, nur das aus Codein bei $206^{\circ}$

1 Liebig Ann. 222, 232.

2 Beim Codein erhielten wir in sehr kleiner Menge auch gelbe nicht hygroskopische Täfelchen, die unter dem Mikroskop Ähnlichkeit mit dem Chloroplatinat des Methylamins zeigten. 
1. $0.2448 g$ gaben $0.0899 g \mathrm{Pt}$

2. $0.2265 \mathrm{~g}$ gaben $0.1100 \mathrm{~g} \mathrm{CO}, 0.0702 \mathrm{~g} \mathrm{H} \mathrm{H}_{2} \mathrm{O}$ und $0.0830 \mathrm{~g} \mathrm{Pt}$

3. $0.1848 g$ gaven $0.0907 g \mathrm{CO}_{2} .0 .0569 g \mathrm{H}_{2} 0$ und $0.0687 \mathrm{gt}$
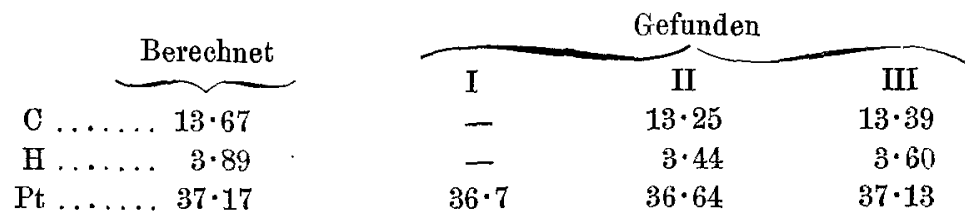

Es kann also nicht daran gezweifelt werden, dass das Äthylmethylamin blos durch Einwirkung des Ätzkali's auf Morphin (und auf Codein) gebildet wird, dass also das Morphin nebst einer Methyl- auch eine Äthylgruppe und beide sehr wahrscheinlich an Stickstoff gebunden enthält.

Ebenso auffallend wie die Abweichung zwischen den Resultaten von L. Knorr und den unsrigen ist der schon früher bemerkte Umstand, dass wir nur etwa die Hälfte des Morphins zu spalten vermochten, während die andere im wesentlichen blos oxydirt wird.

Da wir unsere Untersuchung fortsetzen, enthalten wir uns heute jeder Vermuthung, in welcher Art diese verwirrenden Thatsachen erklärt werden könnten.

Wir fügen nur noch zu, dass bei unseren Versuchen zwei Morphinsorten verschiedener Provenienz das gleiche Verhalten zeigten, dass beide die charakteristischen Morphinreactionen gegeben baben, die eine auch in Morphinmethyljodid iibergeführt wurde, das alle bekannten Eigensehaften besass, dass sich aber bei der Darstellung anderer Morphinderivate Schwierigkeiten einstellten, die seinerzeit auf Zufälligkeiten geschoben wurden. $\mathrm{Ob}$ jenen eine Bedeutung zuzuschreiben ist, müssen weitere Versuche lehren.

$\mathrm{Zn}$ einerZeit in der wir der Meinung waren, das Amin aus Morphin wäre Isopropylamin, haben wir das Verhalten von normalen Propylamin gegen alkoholische Kalilauge studirt. Dabei fanden wir, dass es unter genau denselben Bedingungen erhitzt wie die äquivalente Menge von spaltbarem Morphin, unverändert bleibt. 